\title{
Habilidades Sociales e Intolerancia a la Frustración en adultos
} argentinos

Social Skills and Intolerance to Frustration in Argentinian adults Recepción del artículo: 09-02-2021 | Aceptación del artículo: 11-06-2021

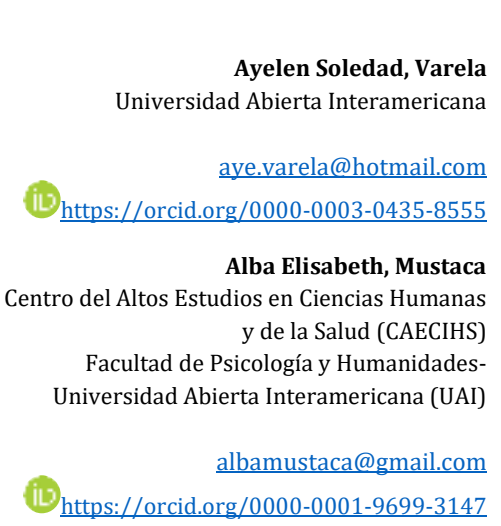

Para referenciar este artículo:

Varela, A. S. y Mustaca, A. E. (2021).

Habilidades Sociales e Intolerancia a la Frustración en adultos argentinos. Revista ConCiencia $E P G, 6(2), 98-116$. https://doi.org/10.32654/CONCIENCIAEP G.6-2.7

\section{Resumen}<smiles>c1ccccc1</smiles>

uando una persona transmite de manera hábil ideas o emociones distintas a la de su interlocutor de manera efectiva se puede afirmar que posee buenas habilidades sociales (HHSS). A la vez, experimenta emociones negativas cuando no logra que las cosas sean como las que esperaba; esta violación de las expectativas se la conoce como frustración. Una alta tolerancia a la frustración y adecuadas HHSS se asociaron a un mayor bienestar psicológico. Esta investigación tuvo como objetivos evaluar diferencias sociodemográficas en las HHSS y en la intolerancia a la frustración (IF) y las relaciones entre ellas con la hipótesis que estarán inversamente correlacionadas. La muestra fue de 100 adultos argentinos. Se utilizaron la Escala de Intolerancia a la Frustración (EIF), la Escala de habilidades sociales (EHS) y un cuestionario sociodemográfico. En la EHS los participantes de nivel universitario tuvieron puntuaciones más altas que los de educación menor y los varones, puntajes mayores respecto de las mujeres en Autoexpresión. Las correlaciones significativas entre la EHHS y la EIF oscilaron entre - .44 a - .22. Un análisis de regresión tomando como variable dependiente a IF total y como independientes a las dimensiones de EHS arrojaron un $\mathrm{r}$ cuadrado = $.29)$.

Palabras Clave: habilidades sociales, intolerancia a la frustración, adultos, género, nivel educativo, edad. 
Revista ConCiencia EPG-Vol. 6- № 2 Julio - Diciembre

2021 ISSN digital: 2517-9896

hitps://doi.org/10.32654/CONCIENCIAEPG.6-2.7

\section{Abstract}

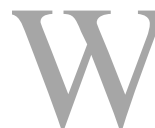

hen a person skillfully conveys ideas or emotions different from that of the interlocutor effectively, it can be said that they have good social skills (HHSS). At the same time, he experiences negative emotions when he does not get things to be as he expected; This violation of expectations is known as frustration. A high tolerance for frustration and adequate HHSS were associated with greater psychological well-being. This study aimed to evaluate sociodemographic differences in the HHSS and in frustration intolerance (FI), and the relationships between them with the hypothesis that they will be inversely correlated. The sample consisted of 100 Argentine adults. The Frustration Intolerance Scale, the Social Skills Scale and an ad-hoc sociodemographic questionnaire were used. In the Social Skills Scale the university-level participants had higher scores than those with lower education and the men had higher scores compared to the women in Self-expression. The significant correlations between EHHS and EIF ranged from - .44 to - .22. A regression analysis taking the total IF as the dependent variable and the EHS dimensions as independent variables yielded and $r$ squared $=.29$ ).

Key Words: social skills, intolerance to frustration, adults, gender, educational level, age.

\section{Introducción}

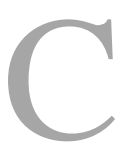

uántas veces hemos conocido personas que en el plano social se desempeñan con fluidez, seguridad, carisma y simpatía, saben poner límites sin ser agresivos y no someterse a ciertas situaciones que le provocan malestar? Estos individuos poseen facilidad en la vinculación interpersonal; en términos psicológicos se los podría clasificar como que poseen muy buenas habilidades sociales (HHSS).

Gismero González (2002) define a las HHSS como: ..."el conjunto de respuestas verbales y no verbales, parcialmente independientes y situacionalmente específicas, a través de las cuales un individuo expresa en un contexto interpersonal sus necesidades, sentimientos, preferencias, opiniones $\mathrm{o}$ derechos sin ansiedad excesiva y de manera no aversiva, respetando todo ello en los demás, que trae como consecuencia el autoreforzamiento y maximiza la probabilidad de conseguir refuerzo externo" (p.14). De un modo similar, Caballo (2007) las concibe como un conjunto de conductas emitidas por un individuo en un contexto interpersonal en las que expresa sentimientos, actitudes, deseos, opiniones o derechos de un modo apropiado para la situación, respetando a su vez esas conductas en los demás.

Las personas que obtienen puntuaciones altas en HHSS se relacionan con variables positivas en su vida. Incluyen el éxito en los negocios (Baron \& Tang, 2009), logros académicos (Caemmerer \& Keith, 2015), protegerse del bullying en 
Revista ConCiencia EPG-Vol. 6- № 2 Julio - Diciembre 2021 ISSN digital: 2517-9896

adolescentes (Polan, Sieving, \& McMorris, 2013), felicidad y calidad de la amistad (Demir, Jaafar, Bilyk, \& Ariff, 2012), satisfacción con sus matrimonios (Villa \& Del Prette, 2013) y confianza interpersonal (Mortenson, 2009), entre otros. Además, cuando se enfrentan a eventos estresantes, las personas con buenas HHSS recurren con efectividad en busca de apoyo en amistades o redes sociales (Mortenson, 2009; Nilsen et.al., 2013) que amortiguan los efectos negativos del estrés (Cohen, Sherrod \& Clark, 1986). En cambio, los déficit en HHSS se asocian a una serie de problemas que incluyen agotamiento laboral (PereiraLimaa \& Loureiroa, 2015), participación en hechos violentos (Polan \& cols, 2013), tendencia tomar decisiones que tienen riesgos sexuales (Curran et al., 2016; Wright et.al, 2010); uso compulsivo de internet (Caplan, 2005; Harfuch et. al, 2010), ansiedad (Monti, 1984, Segrín, 2017, Sorrenti, 2000), esquizofrenia (Brüne et.al, 2011), depresión (Kurimoto, Lueboonthavatchai \& Maes, 2020; Segrin \& Flora, 2000), y mayor probabilidad de estar socialmente aislados y solitarios (Jin \& Park, 2012), entre otros.

Respecto de las comparaciones de la HHSS en función del género, los resultados no son del todo consistentes. Algunos estudios mostraron que las mujeres adultas son más habilidosas en la expresión de sentimientos positivos, mientras que los hombres tienen menor dificultad para expresar sentimientos negativos, hacer peticiones y ser más asertivos en situaciones laborales (Gambrill \& Richey, 1975); otros, que los hombres son más activos en el ámbito social y las mujeres más dependientes y conformistas (e.g.,
Furnham \& Henderson, 1984; Toussaint \& Webb, 2005). Denis, Hamarta \& Ari (2005) hallaron que las mujeres suelen puntuar más en expresividad y sensibilidad emocional y en HHSS que los varones. Caballo et.al. (2014) encontraron que los varones puntuaron más que las mujeres en Hablar o actuar en público/Interaccionar con superiores, Interaccionar con personas que me atraen e Interaccionar con desconocidos, y menos en Pedir disculpas/Reconocer errores propios con tamaños del efecto medianos. García et. al. (2014) hallaron en estudiantes universitarios argentinos que los hombres puntuaron más alto que las mujeres en habilidades para el abordaje afectivo sexual, y más bajo en habilidades conversacionales, de oposición asertiva y en empatía y expresión de sentimientos positivos, con tamaños del efecto bajos en todos ellos. Sin embargo, Salavera, Usan \& Jarie (2017) no hallaron diferencias de género en las HHSS en adolescentes españoles. Los mismos autores hallaron que las mujeres puntuaban más alto en Expresión de desaprobación, con un tamaño del efecto mediano, $y$ más bajos en Decir no e interrumpir interacciones, con un tamaño del efecto bajo (Salavera, Usan, \& Jarie,2020). Sin embargo, Machado, Souza Alves y Fagundes (2020) no hallaron diferencias en las HHSS entre estudiantes en función del género. Estas incongruencias pueden deberse a los diferentes instrumentos usados, a las muestras o a los años de publicación, aunque Caballo (2007) infiere la existencia de diferencias en que los hombres tendrían menos dificultades para concertar citas o llevar a cabo comportamientos asertivos efectivos, y las mujeres, y más competencia 
Revista ConCiencia EPG-Vol. 6- № 2 Julio - Diciembre 2021 ISSN digital: 2517-9896

en la expresión de sentimientos positivos y empatía. Respecto de la empatía, muchos estudios indican que es mayor en las mujeres, y esto se puede manifestar en las HHSS (e.g. Hojat et.al., 2020).

No se hallaron trabajos que comparen las HHSS en función de la edad y fueron muy escasos los que consideraron el nivel educacional ya que se utilizaron muestras en su mayoría homogéneas en esas dos variables. Respecto de la educación y el contexto, Contini, Lacunza y Esterkind (2013) evaluaron las HHSS en adolescentes de 11 y 12 años de Tucumán (Argentina), según su contexto (urbanorural) y su nivel socioeconómico (NES). Los adolescentes urbanos de NES alto tuvieron más consideración hacia los demás que los urbanos y rurales con NES bajo, aunque los adolescentes rurales, con independencia del NES, se percibieron con más liderazgo que sus pares urbanos; a la vez las mujeres rurales, a independencia del NES, presentaron mayor ansiedad social respecto a sus pares mujeres y varones rurales y urbanos.

El adiestramiento en HHSS se utiliza en distintos ámbitos. Rivera et.al (2019) mostraron que el entrenamiento en HHSS en estudiantes universitarios produjo un aumento en esa habilidad comparando los resultados antes y después de la intervención. En el mismo sentido, Cárdenas \& Huaire Inacio (2018), evaluaron antes y después de un entrenamiento en HHSS a un grupo de estudiantes de Educación Física de la Universidad Nacional de Educación Enrique Guzmán y Valle comparando los resultados con un grupo control sin tratamiento.
Comprobaron un mejoramiento en la conducta prosocial de los estudiantes tratados. Gutiérrez Carmona \& Expósito López (2015), también en el contexto universitario, hallaron que el entrenamiento en HHSS mejoró el autoconcepto y el manejo en las dificultades interpersonales. En la escuela primaria, García Esparza y Méndez Sánchez (2017) entrenaron en HHSS en ocasiones específicas a los alumnos con éxito. En áreas de la salud, Sánchez y Rubio (2001) mejoraron las HHSS en enfermeras comparando el tratamiento con un grupo control. En el ámbito clínico, el entrenamiento en HHSS mejoró la comunicación en niños autistas (Koegel \& Frea, 1993), en adolescentes con ansiedad social (Olivares-Olivares, Ortiz-González, \& Olivares, 2019), y en adultos con discapacidad intelectual con un diseño antes después con un grupo control sin tratamiento (Sequera et.al. 2016) y contribuyó prevenir el consumo de alcohol en adolescentes (Senra Varela, 2010). Dryburgh et. al., 2020), en una revisión sistemática mostraron la eficacia del entrenamiento en HHSS en jóvenes con problemas clínicos.

Caballo (2007), afirma que las HHSS están determinadas por una serie compleja de variables. Las clasifica en elementos de tres componentes principales: conductuales (comunicación no verbal y verbal, aspecto físico, contexto, lenguaje, etc.), fisiológicos (tasa cardíaca, respiración, respuesta galvánica de la piel, etc.) y cognitivos (percepción del ambiente y variables cognitivas individuales). Los más estudiados fueron los elementos conductuales y cognitivos. En esta 
Revista ConCiencia EPG-Vol. 6- № 2 Julio - Diciembre 2021 ISSN digital: 2517-9896

presentación interesa enfatizar el último, que incluyen, entre otros, las creencias irracionales y las expectativas. Por ejemplo, la creencia que los demás deben comportarse como nosotros esperamos generan expectativas que en muchos casos no se cumplen y pueden provocar efectos de frustración intensos y de larga duración que alteran las relaciones interpersonales. Caballo (2007, Tabla, 3.5, p. 104) incluye entre los componentes cognitivos que se relacionan con efectivas HHSS la "tolerancia a los conflictos" y "expectativas más precisas acerca la otra persona", aunque aclara que no fueron estudiados respecto de las HHSS. Sin embargo, muchos de los entrenamientos en HHSS incluyen el desarrollo de la tolerancia a la frustración y detectar creencias irracionales (Caballo, 2007). Esto sugiere la necesidad de evaluar las relaciones entre HHSS y tolerancia a la frustración.

Amsel (1992) define la frustración como un estado emocional que se produce ante la disminución de la cantidad o calidad de un reforzador esperado en presencia de una expectativa de recompensas de mayor magnitud. Fue muy estudiada con modelos animales y humanos desde principios del siglo XX, lo que permitió evaluar sus efectos y los mecanismos involucrados. Las respuestas conductuales, fisiológicas y los mecanismos neurales que provoca la frustración de modo inmediato son similares al castigo, la ansiedad, al miedo y al dolor sensorial y provoca conductas de agresividad, enojo, escape, disminución de la autoestima, alteraciones cognitivas, etc. (Mustaca, 2013, 2018).

La intensidad de la frustración depende de factores genéticos, cognitivos, contextuales, motivacionales y de aprendizaje (e.g., Cuenya et.al, 2012, Cuenya, Kamenetzky \& Mustaca, 2014). A mayor distancia entre lo que se espera y lo que se recibe, mayor será la intensidad y duración de los estados frustrantes (e.g., Mustaca, 2018). Coincidiendo con las investigaciones básicas, Ellis (1979) consideró que una alta Intolerancia a la Frustración (IF) y al malestar, y una baja autoestima están fundadas en creencias irracionales (ej. expectativas altas respecto de los demás y de sí mismo que no se cumplen) y son origen de trastornos psicológicos.

La alta IF, al igual que las HHSS deficientes, se asociaron a bajo bienestar psicológico y a patologías. Correlacionó positivamente con procrastinación (ChihHung Ko et al, 2008), con adicción a internet (Harrington, 2005b), autoestima baja (Harrington, 2005a, y Medrano, Franco \& Mustaca, 2018), con la agresividad y la regulación emocional desajustada en adultos (Medrano et.al. 2019) y en adolescentes (Begoña Ibañez, Franco \& Mustaca, 2018) y con un bajo éxito académico (e.g. Zhou, Main, \& Wang, 2010, Butterfield,1962). Una alta IF se relacionó con patologías como la depresión, ansiedad y hostilidad (e.g. Jibeen, 2013), el control de los impulsos en el trastorno límite de la personalidad $y$ en las adicciones (Harrington, 2015a) y con alto neuroticismo (Alba, Gianquino, \& Mustaca, 2020). Por otra parte, Ramirez-Castillo et.al.(2019) mostraron que la tolerancia a la frustración junto con la personalidad y el apego son factores a tener en cuenta en los tratamientos para los problemas de adicción. Veijalainen et.al. (2019) 
Revista ConCiencia EPG-Vol. 6- № 2 Julio - Diciembre 2021 ISSN digital: 2517-9896
https://doi.org/10.32654/CONCIENCIAEPG.6-2.7

mostraron que niños finlandeses de 5 a 7 años con una mejor autorregulación tuvieron más perseverancia en una situación frustrante; Wilde (2012) halló que la tolerancia a la frustración explica el $23 \%$ de la varianza en el éxito académico, y Mustaca (2020) informó que una baja IF regulaba, aunque débilmente, los efectos emocionales en lectores de viñetas sobre ostracismo.

Respecto de la IF y el género, Ko et.al. (2008) y Begoña Ibañez et.al, (2018) en adolescentes mostraron que las mujeres tuvieron mayores puntajes que los hombres en Intolerancia emocional, Demanda de derechos y total de IF. Sin embargo, Alba et. al. (2020) no hallaron diferencias significativas en una muestra en función del género. En función de la edad, participantes de 14 a 17 años obtuvieron puntajes más altos que los de 18 a 28 años, lo mismo que a mayor edad menor IF en una muestra de 800 participantes argentinos (datos no publicados). Thompson (1990), Gross y Thompson (2007) y Capella y Mendoza (2011) mostraron también que, a mayor edad, mayor tolerancia a la frustración. Sin embargo, otras investigaciones no hallaron diferencias significativas en relación a la edad (e.g. Alba et.al. 2019, Begoña y cols. 2018). La disminución de la IF según la edad coincide con la teoría de la frustración, que predice una mayor tolerancia y persistencia ante la frustración teniendo experiencias que varían entre la comprobación o violación las expectativas creadas, propias del desarrollo vital humano.

En relación al nivel educativo, en adolescentes se mostró que los que tenían nivel secundario incompleto obtuvieron mayor puntaje que los de secundario completos en los factores Demanda de Derechos, Intolerancia a la Incomodidad y EIF Total y que a mayor nivel educativo menor IF en adultos (datos no publicados).

En cuanto a las relaciones entre las HHSS y la IF, se halló una sola investigación que asoció ambas variables. Santos Mello y Benevides Soares (2014) hallaron una correlación positiva entre resistencia a la frustración y HHSS en una muestra de 36 estudiantes de primer año y 37 egresados de la carrera de medicina de una universidad de Brasil.

Las investigaciones realizadas de las HHSS y la IF coinciden que adecuadas HHSS y una menor tolerancia a la frustración lleva a un mayor bienestar y viceversa; sin embargo, sólo un trabajo ha mostrado una correlación entre ellas en una pequeña muestra de médicos. Por otra parte, algunas intervenciones para mejorar las HHSS, tanto clínicas como educacionales, incluyen técnicas para adquirir mayor tolerancia a la frustración y modificar creencias irracionales. Aunque por razonamiento lógico se puede hipotetizar que ambas variables están relacionadas, y que se hallarán correlaciones significativas e inversas entre la IF y las HHSS, es la función de la ciencia corroborarlo con evidencia. La siguiente investigación tuvo como objetivos aumentar el conocimiento de las relaciones entre HHSS y la IF en adultos y evaluar ambas variables en función del género, la edad y el nivel educativo. Estos resultados contribuirán a agregar información sobre las vinculaciones entre HHSS e IF; a nivel aplicado permitirá convalidar indirectamente los métodos empleados

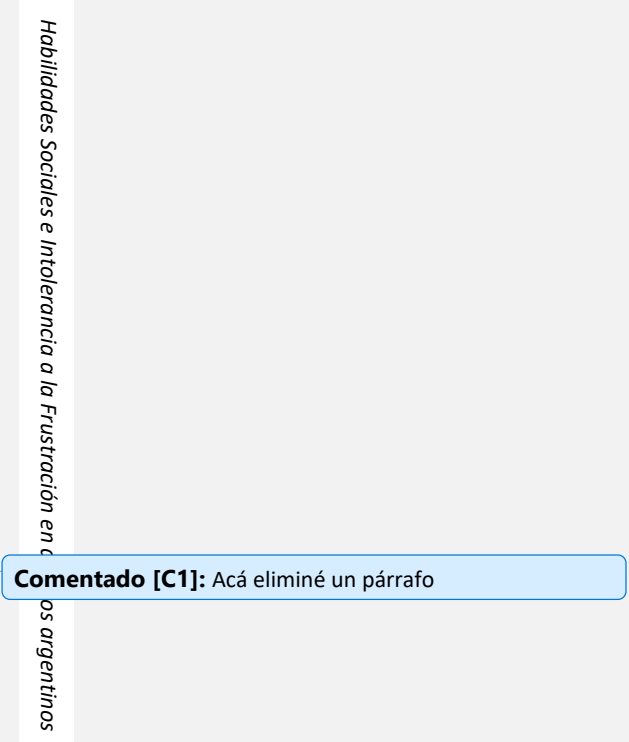

Comentado [C2]: Este párrafo lo cambie bastante 
Revista ConCiencia EPG-Vol. 6- № 2 Julio - Diciembre 2021 ISSN digital: 2517-9896

para mejorar las HHSS que incluyen técnicas para aumentar la tolerancia a la frustración tanto en ámbitos clínicos como educacionales.

\section{Método}

Participantes. Se tomó una muestra no probabilística reclutados con el método de bola de nieve de 100 adultos residentes en Argentina durante 2018. Se excluyeron cuestionarios incompletos y participantes que estaban en tratamiento psiquiátrico. El $34 \%$ residía en la Ciudad de Buenos Aires y el 66\%, en el Gran Buenos Aires. El 63\% eran mujeres, el $36 \%$, varones y el $1 \%$ (n= 1) se identificó como "otros". La edad varió entre 22 a 63 años (promedio=37, SD=9.7). En cuanto al nivel educativo, 3 pertenecían a un nivel primario incompleto $(n=1)$ o completo $(\mathrm{n}=2) ; 27$, a secundario incompleto $(n=5)$ o completo $(n=22) ; 13$, terciario incompleto $(\mathrm{n}=7)$ o completo $(n=6)$ y 57 , universitario incompleto $(n=36)$ o completo $(\mathrm{n}=21)$.

Instrumentos. Escala de habilidades sociales (EHS, Gismero González, 2002). Tiene 33 reactivos con 4 alternativas de respuestas que van desde A (no se parece en nada a mí) hasta $\mathrm{D}$ (se parece mucho a $\mathrm{mi}$ ). A mayor puntaje mayor capacidad en HHSS. Presenta seis dimensiones y una puntuación total: autoexpresión en situaciones sociales (AUOTEX), defensa de los propios derechos como consumidor (DEF), expresión de enfado o disconformidad (ENF), decir no y cortar interacciones (NO), hacer peticiones (PETI)e iniciar interacciones positivas con el sexo opuesto (INI). La escala presenta una confiabilidad total de $\alpha=$.88. La presente muestra arrojó un $\alpha=.82$.

Escala de Intolerancia a la Frustración (EIF, The Frustration Discomfort Scale, Harrington, 2005, adaptación en Argentina de Medrano et al, 2018). Posee 17 ítems con una escala Likert de 5 categorías que van desde "No es nada característico de mí" (1), hasta "Es muy característico de mí" (5). Posee 4 factores y una puntuación total (IFT). A mayor puntaje, mayor IF. El factor Intolerancia a la Incomodidad (INCO) hace referencia a las creencias de que la vida debería ser fácil, cómoda y libre de problemas (ejemplo: "No soporto hacer tareas que me parecen demasiado difíciles"). El factor Demanda de Derechos (DE) refiere a las creencias de que los deseos personales deben ser cumplidos $y$ que las otras personas deben complacer y no frustrar esos anhelos, (ejemplo: "No soporto que otra persona actúe en contra de mis deseos"). El factor Intolerancia Emocional (EMO) hace referencia a la intransigencia a sentir angustia $o$ al malestar emocional, (ejemplo: "No tolero sentir que me estoy volviendo loco). El factor Intolerancia al alcance de Logros (LO) son afirmaciones sobre sentimientos de decepción vinculados con una tarea o a un rendimiento óptimo (ejemplo: "No tolero bajar mis estándares, aun cuando sé que sería más útil hacerlo". La confiabilidad fue de un $\alpha=.61$ para INCO, $\alpha=.76$ para DE, $\alpha$ $=.78$ para EMO y $\alpha=.55$ para LO y $\alpha=.84$ para la IF total (IFT). La presente muestra obtuvo un $\alpha=.79$ para INCO, $\alpha=.87$ para DE, $\alpha=.86$ para EMO, $\alpha=0,80$ para LO y $\alpha=$ .92 para la IFT. 
Revista ConCiencia EPG-Vol. 6- № 2 Julio - Diciembre

2021 ISSN digital: 2517-9896

https://doi.org/10.32654/CONCIENCIAEPG.6-2.7

Cuestionario sociodemográfico adhoc. Indagó sobre edad, género, residencia, estado civil, ocupación y nivel educativo.

Procedimiento. La administración de los cuestionarios se realizó de manera presencial. Se informó a los participantes que la investigación era anónima, voluntaria y que sólo se utilizaría con fines académicos; luego de aceptar su participación y firmar un consentimiento informado se les presentaron los cuestionarios. Este estudio fue aprobado por el comité de ética de la Universidad Abierta Interamericana.

Análisis de resultados. Los datos fueron analizados con el programa estadístico de IBM SPSS, versión 21. La prueba de normalidad arrojó que las escalas no cumplían con los criterios establecidos para utilizar pruebas paramétricas. Como análisis complementario, la variable edad se dividió en dos grupos, tomando la mediana (35 años) como punto de corte, uno con personas de entre 22 a 35 años y el segundo, con participantes de 36 a 63 años.
El nivel educativo se recodificó quedando formado por 5 grupos, 1) no asistió, 2) primario, 3) secundario, 4) terciario y 5) universitario. Debido al escaso número de los grupos 1 y 2 , los análisis inferenciales se realizaron entre los grupos 3,4 y 5 . Se estableció como criterio de significación un $\mathrm{p}<.05$ y el tamaño del efecto se evaluó con la fórmula: $r=\mathrm{Z} / \sqrt{ } \mathrm{N}$ ).

\section{Resultados}

Estadísticos descriptivos. La Tabla I y II muestran los estadísticos descriptivos de los dos cuestionarios de la muestra. Los promedios obtenidos en todas las dimensiones de la EHS y de la EHS total están aproximadamente dentro del percentil 50 establecido en la muestra de Ruiz Alva y Vallejo de Trujillo (2006) en una versión española. En cuanto a la EIF, los promedios de las dimensiones y de la IF total son más bajos, aunque están encuadrados dentro del primer desvío estándar de la muestra presentada por Medrano \& cols. (2018).

Tabla 1

Estadísticos descriptivos de la EHS

\begin{tabular}{lcccc}
\hline \multicolumn{5}{c}{ Estadísticos descriptivos de EHS (n=100) } \\
\hline Factores de EHS & Media & Mínimo & Máximo & Desv. Típico \\
\hline Autoexpresión & 23.2 & 10 & 30 & 4.1 \\
Defensa de derechos & 14.3 & 6 & 20 & 3.1 \\
Expresión de enfado & 11.3 & 5 & 16 & 2.6 \\
Decir No & 18.1 & 10 & 24 & 3.6 \\
Hacer peticiones & 13.5 & 8 & 20 & 2.3 \\
Iniciar interacciones positivas & 12.6 & 7 & 20 & 2.8 \\
EHS Total & 93 & 57 & 119 & 13.1 \\
\hline
\end{tabular}

Tabla 2

Estadísticos descriptivos de la EIF (n=100) 
Revista ConCiencia EPG-Vol. 6- № 2 Julio - Diciembre

2021 ISSN digital: 2517-9896

https://doi.org/10.32654/CONCIENCIAEPG.6-2.7

\begin{tabular}{lcccc}
\hline \multicolumn{1}{c}{ EIF } & Media & Mínimo & Máximo & Desv. Típico \\
\hline Incomodidad & 6.6 & 3 & 15 & 2.9 \\
Derecho & 15.9 & 6 & 30 & 6.3 \\
Emocional & 12.6 & 5 & 25 & 5.3 \\
Logros & 6.5 & 3 & 15 & 3.3 \\
IF Total & 41.6 & 17 & 78 & 15.1 \\
\hline
\end{tabular}

HHSS e IF en función del género, edad y nivel educativo

Los factores de la EIF y IF Total no presentaron diferencias significativas en género y nivel educativo. Respecto de la edad, no se hallaron correlaciones significativas en ninguna de las dos escalas. En cambio, la EHS las tuvo en función del género y del nivel educativo. En relación al género, los varones presentaron puntajes mayores respecto de las mujeres en Autoexpresión ( $\mathrm{Z}=-3,141, \mathrm{p}<.002, \mathrm{r}=.32)$. Se evaluaron tres grupos en función del nivel educativo: 1) secundario, 2) terciario y 3) universitario, tanto completados o no $(\mathrm{n}=96)$, porque la muestra del nivel primario fue escasa $(n=4)$. La prueba Kruskal -Wallis arrojó diferencias significativas en Autoexpresión (Chi Cuadrado $=9.12, \mathrm{p}<.01, \mathrm{r}=.93$ ), Enfado (Chi Cuadrado $=12.03, \mathrm{p}<.002, \mathrm{r}=1.22$ ), Decir no (Chi cuadrado=10.88, $\mathrm{p}<004, \mathrm{r}=1.11$ ), Iniciar conversaciones con el sexo opuesto (Chi Cuadrado= 16.52, $\mathrm{p}<.0001, \mathrm{r}=1.68$ ), $\mathrm{y}$ EHS Total (Chi Cuadrado $=14.2, \mathrm{p}<.001$, $r=1.45$ ). Para evaluar entre cuáles de los tres grupos se hallaban las diferencias, se utilizó la prueba U-Mann-Whitney tomando dos grupos por vez. Los participantes con nivel secundario puntuaron más alto que los del terciario $(\mathrm{n}=40)$ en Enfado $(\mathrm{Z}=-2.60$, $\mathrm{p}<.009, \mathrm{r}=.41)$, Decir No $(\mathrm{Z}=-2.29, \mathrm{p}<.02$, $\mathrm{r}=.36)$ y EHS Total $(\mathrm{Z}=-2.05, \mathrm{p}<.04, \mathrm{r}=.32)$. Los sujetos del nivel universitario puntuaron con mayores HHSS que los del terciario $(\mathrm{n}=70)$ en Autoexpresión $(\mathrm{Z}=-3,66$, $\mathrm{p}<.002, \mathrm{r}=.43)$, Expresión de enfado $(\mathrm{Z}=-$ $3,59, \mathrm{p}<.0001, \mathrm{r}=.43)$, Decir no $(\mathrm{Z}=-3,41$, $0.001, r=.41)$ e Iniciar interacciones con el sexo opuesto $(\mathrm{Z}=-3.602, \mathrm{p}<.0001, \mathrm{r}=.43) \mathrm{y}$ HSS Total ( $\mathrm{Z}=-4.121, \mathrm{p}<.0001, \mathrm{r}=.49)$. Los participantes del nivel universitario puntuaron a la vez más alto que los del nivel secundario en Iniciar interacciones con el sexo opuesto $(\mathrm{Z}=-2.548, \mathrm{p}<.01, \mathrm{r}=.26)$.

\section{Correlaciones entre HHSS e IF}

La Tabla III muestra las correlaciones entre la EHS y la EIF. Se observa que todas fueron inversas, a excepción de una; las significativas fueron entre bajas $y$ medianas. Decir no y cortar interacciones presenta correlaciones significativas bajas en $D E$ (Rho=-.2, p<.04), EMO (Rho=-.25, $\mathrm{p}<.01)$, en INCO (Rho=-.3, $\mathrm{p}<.001)$ y en $I F T$ (Rho=-.3, p<.01); Iniciar Interacciones con el sexo opuesto obtuvo correlaciones bajas y medianas en $D E$ (Rho=-.3, p<.00), EMO (Rho=-.5, p<.000), en INCO (Rho=-.4, $\mathrm{p}<.000)$ y en IFT (Rho=- .4, $p<000)$. Expresión de enfado presentó una correlación baja con INCO (Rho=-.2, p<.05); 
Revista ConCiencia EPG-Vol. 6- № 2 Julio - Diciembre

2021 ISSN digital: 2517-9896

Defensa de los Derechos del consumidor presentó correlaciones medianas en todos los factores de IF: $D E$, LO y EMO (Rho=-3, $\mathrm{p}<.000)$, INCO y en IFT (Rho=-. 4, p<.000). Autoexpresión en situaciones sociales presenta correlaciones entre EMO y INCO y en IFT: Rho=-.23, p<. 02, ; Rho=-.3, p<.001 y Rho=-.3, $\mathrm{p}<.001 \quad$ respectivamente. Finalmente, total de EHS presentó correlaciones con $D E$ (Rho=-.3, p<.001), y con EMO, INCO y en IFT (Rho=-.4, p<.0001).
Un análisis de regresión lineal tomando como variable dependiente a IF total y como variables independientes a las dimensiones de EHS arroja un $\mathrm{R}=.54 \mathrm{r}$ cuadrado $=$.29). Las dimensiones significativas que explican el $29.4 \%$ de la varianza de IF son Defensa de los Derechos del consumidor $(\mathrm{t}=-4.03, \mathrm{p}<.0001)$, Expresión de enfado $(\mathrm{t}=2.56, \mathrm{p}<.01) \mathrm{e}$ Iniciar Interacciones con el sexo opuesto $(\mathrm{t}=$ $-3.35, \mathrm{p}<.001$ ).

Tabla 3

Correlaciones entre la EHS y la EIF $(n=100)$

\begin{tabular}{lllllll}
\hline EHS/EIF & Corr/sig. & DE & Logro & EMO & INCO & TIF \\
\hline Decir NO & Co & -.2 & -.1 & -.25 & -.3 & -.3 \\
& Sig. (bil.) & .04 & 0.3 & .01 & .00 & .00 \\
Int.sexo op. & Co & -.3 & -0.2 & -.5 & -.4 & -.4 \\
Enfado & Sig. (bil.) & .00 & 0.1 & .000 & .000 & .000 \\
& Co & .1 & 0.1 & $-0,2$ & -.2 & -0.1 \\
Def.Derecho & Sig. (bil.) & .68 & .4 & -06 & .05 & .4 \\
& Co & -.3 & -.3 & -.3 & -.4 & -.4 \\
Autoexp & Sig. (bil.) & .000 & .000 & .000 & .000 & .000 \\
& Co & -0.1 & -0.1 & -.23 & -.3 & -.3 \\
Hacer petic. & Sig. (bil.) & .7 & .6 & .02 & .00 & .00 \\
& Co & -0.1 & -0.1 & -0.1 & -0.2 & -0.1 \\
& Sig. (bil.) & .4 & .3 & .3 & .06 & .3 \\
Total EHS & Co & -.3 & -0.1 & -.4 & -.4 & -.4 \\
& Sig. (bil.) & .00 & .2 & .000 & .000 & .000
\end{tabular}

Nota: Rho de Pearson. N=100. INCO= Intolerancia a la incomodidad; DE: derecho; EMO: intolerancia emocional, IFT: Intolerancia a la frustración total. $\mathrm{N}=100$

\section{Discusión y conclusiones}

Tanto las HHSS como la IF fueron estudiadas extensamente y relacionadas 
Revista ConCiencia EPG-Vol. 6- № 2 Julio - Diciembre 2021 ISSN digital: 2517-9896

con diferentes variables. Los entrenamientos en HHSS incluyen en la mayoría de ellos técnicas para la adquisición de una mayor tolerancia a la frustración. Sin embargo, los diseños que mostraron que esos tratamientos no midieron la IF antes y después del mismo. Se halló una sola publicación donde asociaron las HHSS con la tolerancia a la frustración en una pequeña muestra de universitarios. Cada vez más se exige que las prácticas clínicas y educacionales estén basadas en la evidencia, no solamente en el razonamiento lógico o en las creencias. La presente investigación es un aporte teórico y con implicaciones aplicadas. Al evaluar las relaciones entre las HHSS y la IF, prediciendo que ambas correlacionarán de manera inversa, pueden apoyar las intervenciones en HHSS que incluyen aspectos cognitivos; entre ellos, técnicas para aumentar la tolerancia a la frustración. Adicionalmente, se evaluaron ambas variables en relación a la edad, género y nivel educativo.

En función de variables sociodemográficas, la EIF no arrojó diferencias significativas en ninguna de las variables evaluadas. Este resultado coincide con el trabajo de Alba et. al, (2019), aunque otras investigaciones hallaron que las mujeres tienen valores más altos en IF que los varones (ver Introducción) y que a mayor nivel educativo y de edad, la IF disminuye (ver Introducción, Chipea, Negruti \&Trip, 2011), aunque el tamaño del efecto, en aquellas publicaciones que lo informaron, fueron bajos.

En la EHS los varones de esta muestra presentaron valores más altos que las mujeres en el factor autoexpresión con un tamaño del efecto mediano coincidiendo con otros autores (ver Introducción), pero no con otras investigaciones donde hallaron puntajes más altos en las mujeres en algunos factores y menos en otros (ver Introducción, e.g., Sarason et.al. 1985). Se requieren más investigaciones para indagar las relaciones entre HHSS y diferencias de género más actualizadas, ya que la función de la mujer en la sociedad se va acrecentando en estos últimos años con los movimientos feministas, lo que puede ocasionar menos diferencias en las HHSS entre hombres y mujeres.

Los participantes con estudios universitarios puntuaron más alto en HHSS que los de menor nivel educativo con tamaños del efecto mediano. Estos resultados sugieren que el nivel educativo predice un mejor manejo de la frustración y de mejores HHSS. Para seguir indagando en estas relaciones se deberían realizar investigaciones experimentales longitudinales que evalúen los efectos del nivel educativo y la edad en las relaciones entre HHSS y en la IF.

La hipótesis referente a las correlaciones inversas entre HHSS e IF fue confirmada (ver Tabla III). Las que obtuvieron diferencias significativas oscilaron entre Rho $=-.20$ a Rho $=-.45$. Estos datos son similares a los encontrados por Santos Mello y Benevides Soares (2014) utilizando distintas escalas. Los factores interacción con el sexo opuesto y defensa de derechos fueron las que obtuvieron las correlaciones más altas con intolerancia emocional e intolerancia a la incomodidad. Sugiere que las personas que presentan mayor intolerancia emocional y a la 
Revista ConCiencia EPG-Vol. 6- № 2 Julio - Diciembre 2021 ISSN digital: 2517-9896

incomodidad tenderán a tener una mayor dificultad en relacionarse con el sexo opuesto evitando la interacción o comportándose de modo inapropiado; pueden o bien presentar conductas de poca asertividad a la hora de defender sus derechos, ya sea respondiendo de manera agresiva o evitando la confrontación. El análisis de regresión, predice que el entrenamiento en Defensa de los Derechos del consumidor Expresión de enfado e Iniciar Interacciones con el sexo opuesto puede mejorar la IF total, pero más la intolerancia emocional y a la incomodidad. Por otra parte, el adiestramiento en disminuir específicamente la intolerancia emocional y a la incomodidad en los tratamientos en HHSS, potenciaría el mejoramiento en HHSS.

Una de las limitaciones de esta investigación es que la muestra fue no aleatoria y el bajo número de participantes. En el futuro se deberían corroborar estos resultados con un mayor número de participantes en muestras aleatorias y en otros países y contextos.

Al hallarse correlaciones considerablemente altas entre la EIF y las

\section{Referencias}

Alba, L., Giaquinto, L. G. \& Mustaca A. (2020). Dimensiones de la personalidad e Intolerancia a la frustración. Cognición $y$ Comportamiento. ALAMOC, 4, 1525. http://www.alamocweb.org/Revista_Alamoc_4_2020.p df dimensiones de la EHS y que ambos constructos se relacionan con un mayor o menor bienestar psicológico, sugiere que puede existir un factor causal que explique esta relación; un alto puntaje en la dimensión neuroticismo de la personalidad podría ser uno de los factores comunes que explique estas asociaciones. Las futuras investigaciones deberían indagar las asociaciones entre estas tres variables.

En resumen, estos resultados avalan el entrenamiento en HHSS propuesto por Caballo (2007) que contempla desarrollar más tolerancia a la frustración y tener creencias más adecuadas a la realidad entre los componentes cognitivos porque determinan en parte a las HHSS. Se sugiere que en el ámbito clínico y de la educación se evalúen las intervenciones en HHSS que incluya o no el logro de una mayor tolerancia a la frustración y medir la IF antes y después de la intervención para confirmar si disminuye en consonancia con el mejoramiento en las HHSS. Un resultado positivo permitirá comprender mejor los mecanismos involucrados entre ambas variables.

Amsel, A. (1992). Frustration theory: An analysis of dispositional learning and memory. Cambridge, UK: Cambridge University Press.

Baron, R. A., \& Tang, J. (2009). Entrepreneurs' social skills and new venture performance: Mediating mechanisms and cultural 
Revista ConCiencia EPG-Vol. 6- № 2 Julio - Diciembre

2021 ISSN digital: 2517-9896

https://doi.org/10.32654/CONCIENCIAEPG.6-2.7

generality. Journal of Management,

35 ,

282-306.

doi:10.1177/0149206307312513

Begoña Ibañez, M.; Franco, P. \& Mustaca, A. (2018). Intolerancia a la frustración y regulación emocional en adolescentes. En: ConCiencia EPG 3(2):12-33. DOI: https://doi.org/10.32654/

Brüne, M., Schaub,D., Juckel, G., \& Langdon, R. 2011), Social skills and behavioral problems in schizophrenia: The role of mental state attribution, neurocognition and clinical symptomatology. Psychiatry Research, 190(1):9-17 DOI: $10.1016 /$ j.psychres.2010.03.0 15

Butterfield, E.C. (1962). Locus of control, test anxiety, reactions to frustration, and achievement attitudes. Journal of Personality, 32(3), 355-370.

Caballo, V. (2007). Manual de evaluación y entrenamiento de las habilidades sociales. Editorial Siglo XXI, España.

Caballo, V.E., Salazar, Olivares,P., Irurtia, M.J, Olivares,I., \& Toledo, R. (2014). Evaluación de las habilidades sociales: estructura factorial y otras propiedades psicométricas de cuatro medidas de autoinforme. Behavioral Psychology / Psicología Conductual, 22, 3, 375-399.

Caemmerer, J. M., \& Keith, T. Z. (2015). Longitudinal, reciprocal effects of social skills and achievement from kindergarten to eighth grade.
Journal of School Psychology, 53, 265-281. doi:10.1016/j. jsp.2015.05.001

Capella, C. \& Mendoza, M. (2011). Regulación emocional en niños y adolescentes: artículo de revisión. Nociones evolutivas y clínica psicopatológica. Revista Chilena de Psiquiatría y Neurología de la Infancia y Adolescencia, 22(2), 155168.

Caplan, S. E. (2005). A social skill account of problematic internet use. Journal of Communication, 55, 721-736. doi:10.1111/j.14602466.2005.tb03019.x

Cárdenas, A. M. H. C., \& Huaire Inacio, E. J. (2018). Desarrollo de habilidades sociales en contextos universitarios. Horizonte de la Ciencia, 8(14), 123130. https://doi.org/10.26490/unc p.horizonteciencia.

Chih-Hung Ko, M.D., Yu-yu yen,M..D., ChengFang Yen, M.D., Chung-Sheng Chen, M.D \& Shing-Yaw Wang, M.D. (2008). The Association between Internet Addiction and Belief of Frustration Intolerance: The Gender Difference. Cyberpsychology \& Behavior, 11, 3, 60- 72 .

Chipea, F., Negruti, D. \& Trip, S. (2012). Intercultural differences in frustration intolerance. Procedia Social and Behavioral Sciences, 33, 583doi:10.1016/j.sbspro.2012.01.188 
Revista ConCiencia EPG-Vol. 6- № 2 Julio - Diciembre

2021 ISSN digital: 2517-9896

https://doi.org/10.32654/CONCIENCIAEPG.6-2.7

Cohen, S., Sherrod, D., Clark, M. (1986). Social Skills and the StressProtective Role of Social Support. Journal of personality and social psychology, 50, (5):963-73. DOI 10.1037//0022-3514.50.5.963

Contini, E., Lacunza, A. \& Esterkind, A. (2013). Habilidades sociales en contextos urbanos y rurales. Un estudio comparativo con adolescentes. En Psicogente, 16(29), 103-117.

Cuenya, L., Fosacheca, S., Mustaca, A., \& Kamenetzky, G. (2012). Effects of isolation in adulthood on frustration and anxiety. Behavioural Processes, 90, 155-60.

Cuenya, L., Kamenetzky, G., \& Mustaca, A.E. (2014). Early experience and incentive relativity in adulthood.

International Journal of Comparative psychology, 27(3), 474-487.

http://hdl.handle.net/11336/2140 7

Curran, T. M., Monahan, J. L., Samp, J. A., Coles, V. B., DiClemente, R. J., \& Sales, J. (2016). Sexual risk among African American women: Psychological factors and the mediating role of social skills. Communication Quarterly, 64, 536-552. doi:10.1080/01463373.2015.1132 241

Demir, M. S., Jaafar, J., Bilyk, N., \& Ariff, M. R. M. (2012). Social skills, friendship and happiness: A cross-cultural investigation. The Journal of Social
Psychology, 152, 379-385. doi:10.1080/00224545.2011.5914 5

Denis, M., Hamarta, E. \& Ari, R. (2005). An investigation of social skills and loneliness levels of university students with respect to their attachment styles in a sample of turkish students. Social Behavior and Personality, 33(1), pp. 19-32.

Dryburgh, N, Khullar, T., Sandre,S., Persram, J., Bukowski, M. \& Dirks, M. (2020). Evidence Base Update for Measures of Social Skills and Social Competence in Clinical Samples of Youth. Journal of Clinical Child \& Adolescent Psychology, 49, 576-594. https://doi.org/10.1080/1537441 6.2020 .1790381

Ellis, A. (1979). Discomfort anxiety: A new cognitive behavioral construct. Part I. Rational Living, 14, 3-8.

Filippello, P., Harrington,N., Sorrenti, L.,Buzzai, C. \& Costa, L.(2014). The Relationship Between Frustration Intolerance, Unhealthy Emotions, and Assertive Behaviour in Italian Students. Journal of RationalEmotive \& Cognitive-Behavior Therapy, 32, 40-59. DOI: $10.1007 / \mathrm{s} 10942-014-0193-4$

Furnham, A. \& Henderson, M. (1984). Assesing assertiveness: A content and correlational analysis of five assertiveness inventories. Behavioral Assessment, 6(1), pp. 79 88. 
Revista ConCiencia EPG-Vol. 6- № 2 Julio - Diciembre

2021 ISSN digital: 2517-9896

hitps://doi.org/10.32654/CONCIENCIAEPG.6-2.7

Gambrill, E. D., \& Richey, C. A. (1975). An assertion inventory for use in assessment and research. Behavior Therapy, 6(4), 550-561. https://doi.org/10.1016/S00057894(75)80013-X

García Esparza. A. \& Méndez, C. (2017). El entrenamiento en habilidades sociales y su impacto en la convivencia escolar dentro de un grupo de primaria. Revista de Estudios y Experiencias en Educación, 16,151 - 164. doi:10.21703/rexe.201730151164 8 ISSN 0717-6945 ISSN

García, M., Cabanillas, G., Morán, V. \& Olaz, F. (2014). Diferencias de género en habilidades sociales en estudiantes universitarios de Argentina. Anuario Electrónico de Estudios en Comunicación Social "Disertaciones", 7(2), 5. Disponible en la siguiente dirección electrónica:

http://erevistas.saber.ula.ve/index. php/Disertaciones/

Gismero, E. (2002). Escala de Habilidades Sociales. Madrid, TEA Ediciones.

Gross, J. \& Thompson, R. (2007). Emotion regulation: Conceptual foundations. En J. Gross (Ed.), Handbook of Emotion Regulation (3-22). New York: Guilford Press.

Gutiérrez Carmona, M. \& Expósito López, J. (2015).Autoconcepto, dificultades interpersonales, habilidades sociales y conductas asertivas en adolescentes // Self-concept, interpersonal difficulties, social skills \& assertiveness in teenag. Revista Española de Orientación y Psicopedagogía, 26, 42-54. DOI: 10.5944/reop.vol.26.num.2.20 15.15215

Harfuch, M., Pacheco Murguía, M., Lever, P \& Zavala Andrade, D. (2010). La adicción a facebook relacionada con la baja autoestima, la depresión y la falta de habilidades sociales. Psicología Iberoamericana, 18, 6-18. https://psicologiaiberoamericana.i bero.mx/index.php/psicologia/arti cle/view/256

Harrington, N. (2005a). Dimensions of frustration intolerance and their relationship to self-control problems. Journal of RationalEmotive \& Cognitive-Behavior Therapy, 23,1-20.

Harrington, N. (2005b). The Frustration Discomfort Scale: Development and Psychometric Properties. Clinical Psychology and Psychotherapy, 12, 374-387.

Hojat, M., DeSantis, J. Shannon, S., Speicher, M.R. Bragan, L., | Calabrese, L.H. (2020) Empathy as related to gender, age, race and ethnicity, academic background and career interest: A nation wide study of osteopathic medical students in the United States . Medical Education, 54,571-581, DOI: $10.1111 /$ medu.14138

Jibeen, T. (2013). Frustration Intolerance Beliefs as Predictors of Emotional 
Revista ConCiencia EPG-Vol. 6- № 2 Julio - Diciembre

2021 ISSN digital: 2517-9896

https://doi.org/10.32654/CONCIENCIAEPG.6-2.7

Problems in University Undergraduates SP. Journal of Rational-Emotive \& CognitiveBehavior Therapy, 31(1), 25-36. DOI: $10.1007 / \mathrm{s} 10942-012-0154-8$

Jin, B., \& Park, N. (2012). Mobile voice communication and loneliness: Cell phone use and the social skills deficit hypothesis. Journal New Media \& Society, 15, 1094-1111. doi:10.1177/1461444812466715

Ko, C.-H., Yen, J.-Y., Yen, C.-F., Chen, C.-S., \& Wang, S.-Y. (2008). The Association between Internet Addiction and Belief of Frustration Intolerance: The Gender Difference. CyberPsychology \& Behavior, 11(3), 273278. doi:10.1089/cpb.2007.0095

Koegel, R. \& Frea, W. (1993). Treatment of social behavior in autism through the modification of pivotal social. Journal of Applied Behavior Analysis, 26 , 369-377. https://doi.org/10.1901/jaba.1993 .26-369

Kurimoto, P.; Lueboonthavatchai, P.; Maes, M. (2020). Impaired Social Skills as a Key Component of Clinical Depression: Associations with Severity of Illness, Self-Esteem, Family Functional Health Satisfaction, and Personality Features. Preprints, 2020050122 (doi:10.20944/preprints202005.01 22.v1)

Machado, Henrique de Souza Alves, S., \& Fagundes Caetano, P (2020). Relationship between social skills, stress, age, sex, school type and school grade in adolescents. Fractal: Revista de Psicología, 32, 210-217, doi:

https://doi.org/10.22409/1984-

0292/v32_i-esp/39792

Medrano, L. A., Franco, P., \& Mustaca, A. E. (2018). Adaptación argentina de la escala "Escala de intolerancia a la frustración." Behavioral Psychology/Psicología Conductual, 26(2), 303-321.

Medrano, L. A., Franco, P., Flores-Kanter, P. E., \& Mustaca, A. E. (2019). Intolerancia a la frustración y estrategias cognitivas de regulación emocional en la predicción de la agresividad. Suma Psicológica, 26(1), 19-27. https://doi.org/10.14349/sumapsi .2019.v26.n1.3

Monti, P. M. (1984). Multi-modal measurement of anxiety and social skills in a behavioral role-play test: Generalizability and discriminant validity. Behavioral Assessment, 6, 15-25.

Mortenson, S. T. (2009). Interpersonal trust and social skill in seeking social support among Chinese and Americans. Communication Research, 36, 32-53. doi:10.1177/0093650208326460

Mustaca, A. (2013). "Siento un dolor en el alma": ¿metáfora o realidad? Revista Argentina de Ciencias del comportamiento. 5, 2, 47-60. 
Revista ConCiencia EPG-Vol. 6- № 2 Julio - Diciembre

2021 ISSN digital: 2517-9896

https://doi.org/10.32654/CONCIENCIAEPG.6-2.7

Mustaca, A. E. (2018). Frustración y conductas sociales. Avances En Psicología Latinoamericana, 36(1), 65-81.

https://doi.org/10.12804/revistas. urosario.edu.co/apl/a.4643

Mustaca, A. E. (2020). Efectos emocionales y cognitivos de viñetas sobre exclusión social. Conferencia. Actas de Resúmenes de la XVII Reunión Nacional y VI Encuentro Internacional de la Asociación Argentina de Ciencias del Comportamiento RACC, 2020, Suplemento (Mayo), 25-26. https://revistas.unc.edu.ar/index.ph p/racc/issue/view/2105/324

Nilsen, W., Karevold, E., Røysamb, E., Gustavson, K., \& Mathiesen, K. S. (2013). Social skills and depressive symptoms across adolescence: Social support as a mediator in girls versus boys. Journal of Adolescence, 36 , 11-20.

doi:10.1016/j.adolescence. 2 012.08 .005

Olivares-Olivares, P.J., Ortiz-González, P.F. and Olivares, J.(2019). Role of social skills training in adolescents with social anxiety disorder. International Journal of Clinical and Health Psychology,19, 41-48. https://doi.org/10.1016/j.ijchp.20 18.11.0021697-2600/

Pereira-Limaa, K., \& Loureiroa, S. R. (2015). Burnout, anxiety, depression, and social skills in medical residents. Psychology, Health \& Medicine, 20, 353-362. doi:10.1080/13548506.2014.9368 89

Polan, J. C., Sieving, R. E., \& McMorris, B. J. (2013). Are young adolescents' social and emotional skills protective against involvement in violence and bullying behaviors? Health Promotion Practice, 14, 599606.

doi:10.1177/1524839912462392

Ramirez-Castillo D., Garcia-Roda C., Guell F, Fernandez-Montalvo J., Bernacer J. and Morón I. (2019). Frustration Tolerance and Personality Traits in Patients With Substance Use Disorders. Front. Psychiatry 10:42 doi: $10.3389 /$ fpsyt.2019.00421

Rivera, J., Lay, N. Moreno, M. E.; Perez, A. Rocha, G.; Parra, M.; Durán, S.; García. J. Redondo, O.\& Torres Rivas, E. (2019). Programa de entrenamiento para desarrollar habilidades sociales en estudiantes universitarios. Revista espacios, 40, 10 , 10-12. https://www.revistaespacios.com/ a19v40n31/a19v40n31p10.pdf

Ruiz Alva, C. \& Vallejo de Trujillo, C. (2006). Escala de habilidades sociales EHS). En:https://www.academia.edu/10 882960/ESCALA DE HABILIDADE S SOCIALES EHS . extraída el 3 de agosto de 2020.

Sánchez, M.\& Rubio, J. (2001). Entrenamiento en habilidades sociales: un método de enseñanzaaprendizaje para desarrollar las habilidades de comunicación 
Revista ConCiencia EPG-Vol. 6- № 2 Julio - Diciembre

2021 ISSN digital: 2517-9896

https://doi.org/10.32654/CONCIENCIAEPG.6-2.7

interpersonal. Psicothema, 13, 247251

Salavera, C., Usán, P. \& Jarie, L. (2020). Styles of humor and social skills in students. Gender differences. Current Psychology, 1-10. https://doi.org/10.1007/s12144017-9770-x

Salavera, C., Usan, P., Jarie, L. (2017). Emotional intelligence and social skills on self-efficacy in Secondary Education students. Are there gender differences? Journal of Adolescence, 60, 39-46. https://doi.org/10.1016/i.adolesce nce.2017.07.009

Santos Mello, T.V. \& Benevides Suares, A. (2014). Habilidades sociales y frustración en estudiantes de medicina. Ciencias Psicológicas,2, $163 \quad-\quad 172$. https://www.redalyc.org/pdf/459 5/459545413006.pdf

Sarason, B. R., Sarason, I. G., Hacker, T. A., \& Basham, R. B. (1985). Concomitants of social support: Social skills, physical attractiveness, and gender. Journal of Personality and Social Psychology, 49(2), 469480. https://doi.org/10.1037/002 2-3514.49.2.469

Segrin, C. (2000). Social skills deficits associated with depression. Clinical Psychology Review, 20, 379-403. doi:10.1016/S02727358(98)00104-4

Segrin, C. (2017). Indirect Effects of Social Skills on Health Through Stress and
Loneliness, Health Communication, 34,1, 118-124, DOI: 10.1080/10410236.2017.1384434

Segrin, C., \& Flora, J. (2000). Poor social skills are a vulnerability factor in the development of psychosocial problems. Human Communication Research, 26, 489-514. doi:10.1111/j.1468-

2958.2000.tb00766.x

Sequera Fernandez, E., Padilla-Muñoz, E., Chirino Núñez, J.\& Pérez-Gil, L. (2016). Aplicación de un nuevo programa de habilidades sociales a un grupo de adultos con discapacidad intelectual. Revista Española de Discapacidad (REDIS), $4 \quad$ (2), 6380. http://hdl.handle.net/11441/6 0890

Serna Varela, M. (2010). Educar en habilidades sociales para prevenir el abuso del alcohol en la adolescencia Revista Española de Orientación y Psicopedagogía, 21,2, 423-433.

DOI: https://doi.org/10.5944/reop.vol.2 1.num.2.2010.11556

Filippello, P., Harrington,N., Sorrenti, L.,Buzzai, C. \& Costa, L.(2014). The Relationship Between Frustration Intolerance, Unhealthy Emotions, and Assertive Behaviour in Italian Students. Journal of RationalEmotive \& Cognitive-Behavior Therapy, 32, 40-59. DOI: $10.1007 / s 10942-014-0193-4$ 
Revista ConCiencia EPG-Vol. 6- № 2 Julio - Diciembre

2021 ISSN digital: 2517-9896

https://doi.org/10.32654/CONCIENCIAEPG.6-2.7

Thompson, R. (1990). Emotion and selfregulation. En R. A. Thompson (Ed.). Socioemotional development. Nebraska Symposium on Motivation. 36, 367-467. Lincoln: University of Nebraska Press

Torres, A. M. \& Piña, J. A. (2010). Asociación entre variables psicológicas y sociales con la adhesión en personas con diabetes tipo 2 . Terapia psicológica, 28, 1, 45-53.

Toussaint, L. \& Webb, J. (2005). Gender Differences in the Relationship Between Empathy and Forgiveness. The Journal of Social Psychology, 145, 673-685.

Veijalainen, J., Reunamo, J., Sajaniemi, N. \& Suhonen, E., (2019), 'Children's selfregulation and coping strategies in a frustrated context in early education', South African Journal of Childhood Education. 9(1), a724. https://doi.org

10.4102/sajce.v9i1.724

Villa, M. B., \& Del Prette, Z. A. P. (2013). Marital satisfaction: The role of social skills of husbands and wives.

Paidéia, 23, 379-387. doi:10.1590/ 1982-43272356201312

Wilde, J. (2012). The Relationship between Frustration Intolerance and Academic Achievement in College Indiana University East. International Journal of Higher Education, 1, 2, doi:10.5430/ijhe.v1n2p1

Wright,K., Banas, J.,Bessarabova, E. \& Bernard, D.(2010). A Communication Competence Approach to Examining Health Care Social Support, Stress, and Job Burnout Health Worker. Health Communication, 25, 375-382, DOI: $10.1080 / 10410231003775206$

Zhou, Q., Main, A., \& Wang, Y. (2010). The relations of temperamental effortful control and anger/frustration to Chinese children's academic achievement and social adjustment: A longitudinal study. Journal of Educational Psychology, 102(1), $180-$

196. https://doi.org/10.1037/a001 $\underline{5908}$ 